\title{
Frequency Invariant MVDR Beamforming without filters and Implementation using MIMO radar
}

\author{
Piya Pal and P. P. Vaidyanathan \\ Dept. of Electrical Engineering, MC 136-93 \\ California Institute of Technology, Pasadena, CA 91125, USA \\ E-mail: piyapal@caltech.edu, ppvnath@systems.caltech.edu
}

\begin{abstract}
Frequency invariant beamforming with sensor arrays is generally achieved using filters in the form of tapped delay-lines following each sensor. However it has been recently shown that with the help of the rectangular smart antenna array, it is possible to generate frequency invariant beampattern without using filters. In this paper, this frequency invariant beamforming technique is utilized to perform MVDR beamforming in the beamspace by designing frequency invariant beams spanning the desired range of azimuthal angles and optimally combining them. However the performance of the frequency invariant beamformer depends on the number of sensors which could be large for a rectangular array of size $M \times N$. Making use of the virtual array concept used in MIMO radar, a novel method of producing the same frequency invariant beam, using only $M$ transmitting and $N$ receiving antennas, is proposed and a design example is provided to demonstrate the idea.
\end{abstract}

Index Terms - Beamspace, Frequency Invariance, MIMO Radar, MVDR, Virtual Array.

\section{INTRODUCTION}

In array processing of broadband signals, frequency invariance is usually achieved with the help of tapped delay-lines at the output of each sensor. Frequency invariant beamforming has been studied extensively because of the advantages it offers in terms of reduced complexity for adaptive beamforming [3], [8], [9], broadband DOA estimation and so forth [3], [11]. Generally frequency invariant beamforming needs filters at the sensor outputs to compensate for the frequency dependence of the beampattern.

In [4], however, it has been shown that frequency invariant beamforming can be achieved using just one weight per sensor instead of tapped delay-lines, by extending the dimension of the array from linear to rectangular. We review this idea briefly in Section II. The contributions of our paper are twofold, which are discussed in Sections III and IV. In Section III of this paper, we use this idea to the case of beamspace MVDR beamforming. Using a rectangular array of dimension $M \times N$, we generate a set of $P$ fixed frequency invariant beams, specified as functions of azimuthal angle, following the method suggested in [4], and then combine them adaptively, by taking a weighted sum of the beams. These weights can be adapted in real time. Here they are designed so that the resulting beam is optimum in the MVDR sense. It is to be noted that $P$, the number of weights to be adapted, is much less than the total number of sensors $M \times N$ and so it offers reduced complexity in adapting these weights in real time. Moreover, unlike [3], [8] and [9], the generation of the frequency invariant beams is achieved without tapped delayline filters. In [5], adaptive beamforming is also performed using the rectangular array without filters but it uses one weight per sensor. So it requires the adaptation of $M N$ weights whereas our method can perform the adaptation using far fewer number of weights. Also the resulting beampattern in [5] is not guaranteed to be frequency invariant whereas

${ }^{1}$ Work supported in parts by the ONR grant N00014-08-1-0709, and the California Institute of Technology. in our design the final beampattern will always be frequency invariant.

The performance of the frequency invariant beamformers however depends on the size of the array and for a rectangular array, it could mean the use of a large number of sensors. A solution to this is proposed in Section IV by using the virtual array concept prevalent in MIMO radar beamforming. Thus, a novel idea of producing the same frequency invariant beampattern as generated by a rectangular array, using only $M$ transmitting and $N$ receiving antennas, is proposed where the transmitted signals are wideband. The transmitting and receiving arrays are both uniform linear arrays (ULA) and they are along orthogonal directions. We perform the desired beamforming at the receiver using only one weight per sensor and produce the same beampattern, effectively using $M+N$ sensors instead of $M N$ sensors. This is also different from [6] where the beamforming for a wideband MIMO radar is performed by dividing the wideband signal into narrow frequency bins. We provide simulation examples to support our proposed method in Section V.

\section{REVIEW OF FREQUENCY INVARIANT BEAMFORMING USING RECTANGULAR ARRAY}

Consider a uniform rectangular array of size $M \times N$. Each omnidirectional antenna element's position is denoted by $\left(m d_{1}, n d_{2}\right), m=-\frac{M-1}{2}, \cdots, \frac{M-1}{2}, n=-\frac{N-1}{2}, \cdots, \frac{N-1}{2}$, $d_{1}$ and $d_{2}$ being the spacing between elements along the two dimensions respectively. We assume $M$ and $N$ to be odd. Signal received at $(m, n)$ th sensor is multiplied by $C_{m, n}$ and then all these signals are summed up to get the beam. Assuming the wideband signal $s(t)$ to be arriving from an elevation angle $\phi$ and azimuthal angle $\theta$, the signal received at the $(m, n)$ th antenna, after demodulation, and sampling with sampling period of $T_{s}$, is given in the frequency domain as

$X_{m, n}\left(e^{j \omega}, \theta\right)=e^{-j \frac{\hat{\omega}}{c T_{s}}\left(m d_{1} \sin \phi \cos \theta+n d_{2} \sin \phi \sin \theta\right)} S\left(e^{j \omega}\right)$

where $c$ is the propagation velocity and $\widehat{\omega}=\omega+2 \pi f_{c} T_{s}, f_{c}$ being the carrier frequency. Assuming that $\phi \approx 90^{\circ}$ [4], the beam pattern is given by

$H\left(e^{j \omega}, \theta\right)=\sum_{m=-\frac{M-1}{2}}^{\frac{M-1}{2}} \sum_{n=-\frac{N-1}{2}}^{\frac{N-1}{2}} C_{m, n} e^{-j \frac{\hat{\omega}}{c T_{s}}\left(m d_{1} \cos \theta+n d_{2} \sin \theta\right)}$

Suppose we want to generate a desired frequency invariant beampattern $F(\theta)$. Then we require

$$
H\left(e^{j \omega}, \theta\right)=F(\theta), \quad \omega_{l} \leq \omega \leq \omega_{h}
$$

where $\omega_{l}$ and $\omega_{h}$ are the lowest and highest frequencies respectively contained in $S\left(e^{j \omega}\right)$. Denoting $\omega_{1}=\widehat{\omega} \frac{d_{1}}{c T_{s}} \cos \theta$, $\omega_{2}=\widehat{\omega} \frac{d_{2}}{c T_{s}} \sin \theta$ and $G\left(\omega_{1}, \omega_{2}\right)=H\left(e^{j \omega}, \theta\right)$ in (2), we have 
$G\left(\omega_{1}, \omega_{2}\right)=\sum_{m=-\frac{M-1}{2}}^{\frac{M-1}{2}} \sum_{n=-\frac{N-1}{2}}^{\frac{N-1}{2}} C_{m, n} e^{-j m \omega_{1}} e^{-j n \omega_{2}}=F\left(\tan ^{-1}\left(\frac{\omega_{2} d_{1}}{\omega_{1} d_{2}}\right)\right)$ for $\frac{\left(c T_{s}\right)^{2} \omega_{1}^{2}}{d_{1}^{2}}+\frac{\left(c T_{s}\right)^{2} \omega_{2}^{2}}{d_{2}^{2}} \in\left[\left(\omega_{l}+2 \pi f_{c} T_{s}\right)^{2},\left(\omega_{h}+2 \pi f_{c} T_{s}\right)^{2}\right]$ Beyond this range of $\frac{\left(c T_{s}\right)^{2} \omega_{1}^{2}}{d_{1}^{2}}+\frac{\left(c T_{s}\right)^{2} \omega_{2}^{2}}{d_{2}^{2}}, G\left(\omega_{1}, \omega_{2}\right)$ can theoretically take any value without affecting the desired beampattern. We assume it to be a constant value beyond this range. We choose $d_{1}=d_{2}=\frac{\lambda_{\min }}{2}$ to avoid spatial aliasing, $\lambda_{\min }$ being the smallest wavelength in $s(t)$. We determine $C_{m, n}$ by

$$
C_{m, n}=\frac{1}{K L} \sum_{k=0}^{K-1} \sum_{l=0}^{L-1} G\left(\omega_{1 k}, \omega_{2 l}\right) e^{j m \omega_{1 k}} e^{j n \omega_{2 l}},
$$

where $\omega_{1 k}=-\pi+\frac{2 \pi k}{K}, \omega_{2 l}=-\pi+\frac{2 \pi l}{L}$ with $K=2 M$ and $L=2 N$ [10].

\section{BEAMSPACE MVDR BEAMFORMING USING FREQUENCY INVARIANT BEAMS}

In this section we extend the above idea to produce $P$ fixed (non adaptive) frequency invariant orthogonal beams spanning the range of azimuthal angles of interest and then linearly combine them, to produce an MVDR beam. Fig. 1 demonstrates this.

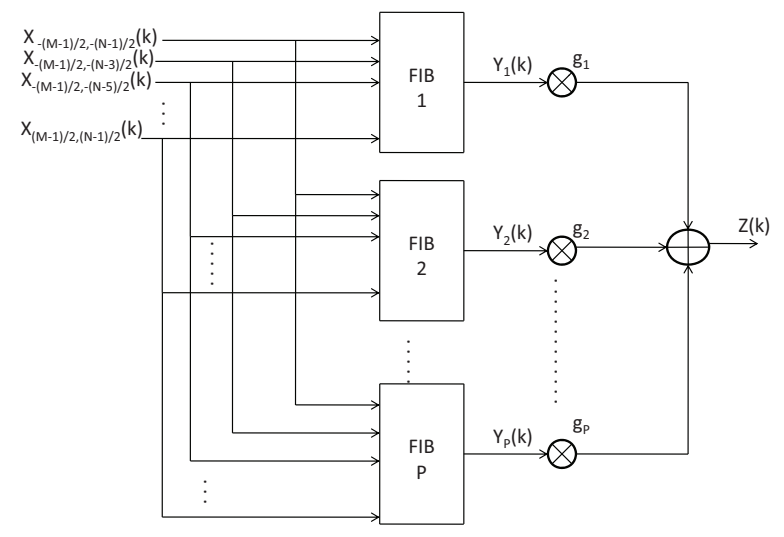

Fig. 1. Block diagram of FIB and beamspace processor.

The sampled signals $x_{m, n}(k) m=-\frac{M-1}{2}, \cdots, \frac{M-1}{2}, n=$ $-\frac{N-1}{2}, \cdots, \frac{N-1}{2}$, from all the $M N$ antennas are fed into each of the $P$ Frequency Invariant Beamformers (FIB) which form the desired $P$ frequency invariant orthogonal beams spanning the beamspace. The $p$ th FIB uses the constant weights $C_{m, n}^{p}$, $m=-\frac{M-1}{2}, \cdots, \frac{M-1}{2}, n=-\frac{N-1}{2}, \cdots, \frac{N-1}{2}$ to form the $p$ th beam $B_{p}(\theta)$ as

$$
\sum_{m=-\frac{M-1}{2}}^{\frac{M-1}{2}} \sum_{n=-\frac{N-1}{2}}^{\frac{N-1}{2}} C_{m, n}^{p} e^{-j m \omega_{1}} e^{-j n \omega_{2}}=B_{p}(\theta)
$$

for $p=1,2, \cdots, P$. In this case, $B_{p}(\theta)$ is chosen to be

$$
B_{p}(\theta)=\frac{1}{P} \frac{\sin \left(\frac{\pi P}{2}\left(\sin \theta-\frac{2 p-P-1}{P}\right)\right)}{\sin \left(\frac{\pi}{2}\left(\sin \theta-\frac{2 p-P-1}{P}\right)\right)}
$$

It is to be noted that these FIBs do not use any tapped delayline and each FIB generates one frequency invariant beam using only one weight per sensor output. For each FIB, $C_{m, n}^{p}$ are determined by the method proposed in Section I. We combine the outputs $y_{p}(k)$, of the $P$ FIBs by multiplying them with respective adaptive weights $g_{p}, p=1,2, \cdots, P$ and taking the sum to get the final output $z(k)$. Consider $I$ broadband signals $s_{i}(k)$ incident on the array at azimuthal angles $\theta_{i}, i=0,1 \cdots, I-1$ with $s_{0}(k)$ being the desired signal. Using (5), the output of the $p$ th FIB is,

$$
y_{p}(k)=\sum_{i=0}^{I-1} B_{p}\left(\theta_{i}\right) s_{i}(k)+\eta_{p}(k)
$$

where $\eta_{p}(k)$ is the noise at the output of $p$ th FIB. Define $\mathbf{y}=\left[\begin{array}{lll}y_{1}(k) & y_{2}(k) \cdots y_{P}(k)\end{array}\right]^{T}$ and $\mathbf{g}=\left[\begin{array}{ll}g_{1} & g_{2} \cdots g_{P}\end{array}\right]^{H}$. Then the final output is given by

$$
z(k)=\mathbf{g}^{H} \mathbf{y}
$$

The SINR maximization can be obtained by minimizing the total variance of $z(k)$ under the constraint that the target response, from look direction $\theta_{0}$ is unity. It can be expressed as the following optimization problem

$$
\min _{\mathbf{g}} \mathbf{g}^{H} \mathbf{R g}
$$

$$
\text { subject to } \mathbf{g}^{H} \mathbf{b}=1
$$

where $\mathbf{R} \triangleq E\left[\mathbf{y y}^{H}\right]$ and $\mathbf{b} \triangleq\left[B_{1}\left(\theta_{0}\right) \quad B_{2}\left(\theta_{0}\right) \cdots B_{P}\left(\theta_{0}\right)\right]^{T}$. This $\mathbf{g}$ is called minimum variance distortionless response (MVDR) beamformer and the well known solution to the above optimization problem is given by [2]

$$
\mathbf{g}=\frac{\mathbf{R}^{-1} \mathbf{b}}{\mathbf{b}^{H} \mathbf{R}^{-1} \mathbf{b}}
$$

This way we can find the optimum weights $g_{p}, p=1,2, \cdots, P$ which will maximize the SINR and will effectively suppress upto $P-1$ jammers arriving from directions other than $\theta_{0}$. We illustrate this in Section $\mathrm{V}$ with simulation examples.

It is to be noted that the FIB processors produce fixed beams and hence can be designed off-line. The actual number of weights to be adapted is $P$ which is far less than the total number of sensors $M N$. This could be achieved because the frequency invariant beamforming and the adaptive beamforming are separately performed in this case, so that even when the signals are wideband, we end up adapting only one constant weight per beam (as the beams are designed to be frequency invariant). This is also fundamentally different from [5] where one weight per sensor is adapted, resulting in a total of $M N$ weights to be adapted. This involves adaptation of a large number of weights in real time compared to our method, when $P \ll M N$. Also, the resulting beampattern in [5] is not necessarily frequency invariant, whereas our method will always produce a frequency invariant beam, as we shall demonstrate in Section V.

\section{IMPLEMENTATION OF THE FIB USING MIMO RADAR VIRTUAL ARRAY CONCEPT}

Although the frequency invariant beamforming method described in Section II uses no filter, the performance of the FIB however depends on the size $M \times N$ of the rectangular array which could mean the use of a large number $M N$ of sensors. So here we propose a modified method to produce a given frequency invariant beampattern in wideband MIMO radar applications, using only $M+N$ sensors which will produce the desired frequency invariant beam as is produced 
by a rectangular array of size $M \times N$ at the receiver. Fig. 2 shows a MIMO radar with $M$ transmitting and $N$ receiving antennas with $d$ as the inter-element spacing. The transmitting and receiving arrays are linear, omnidirectional, colocated and perpendicular to each other. The azimuthal angle, $\theta$ is measured in their plane ( $X Y$ plane) and the elevation angle $\phi$ is measured with respect to the normal to $X Y$ plane. The

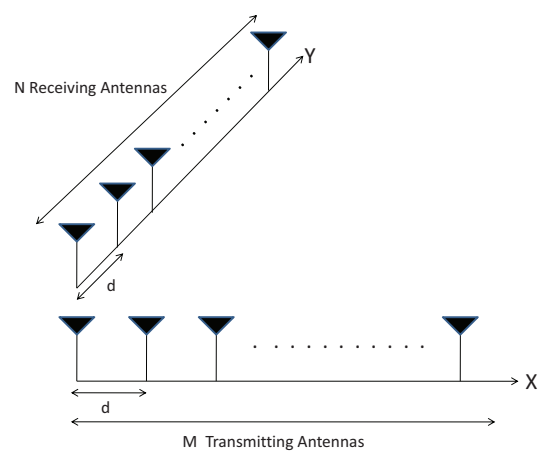

Fig. 2. MIMO radar Transmitting and Receiving Arrays.

transmitted signal of the $m$ th transmitting antenna is given by

$$
s_{m}(t)=\tilde{s}(t) e^{j \Omega_{m} t}
$$

where $\tilde{s}(t)$ is a wideband signal occupying a frequency range from $f_{l}$ to $f_{h}$ with a total bandwidth of $B$. The $\Omega_{m}$ are chosen such that

$$
\Omega_{m+1}-\Omega_{m} \geq 2 \pi B \quad m=0,1, \cdots, M-1 .
$$

This ensures that the signals transmitted from the different transmitted antennas are non overlapping in frequency. At each receiving antenna, we have a bank of $M$ linear phase bandpass filters to separate out the $M$ transmitted signals received at any receiving antenna, retaining their phase information. The $m$ th bandpass filter has a bandwidth of $2 \pi B$ centered at frequency $\Omega_{m}$. Consider a far-field point target at elevation angle $\phi$ and azimuthal angle $\theta$. The aggregate waveform incident on the target is

$$
p(t)=\alpha_{1} \sum_{m=0}^{M-1} s_{m}\left(t-\frac{d}{c} m \sin \phi \cos \theta\right)
$$

where $\alpha_{1}$ is the overall attenuation factor, assumed same for all transmitters [6]. The signal received from the target at the $n$th receiving array is given as $x_{n}(t)=\alpha_{2} p\left(t-n \frac{d}{c} \sin \phi \sin \theta\right)$ for $n=0,1, \cdots, N-1$, where $\alpha_{2}$ depends on the propagation attenuation and target's scatter coefficient [6]. Assuming the bandpass filters are ideal zero phase filters, the target response in the $m$ th bandpass filter of the $n$th receiving antenna is

$$
r_{n, m}(t)=\alpha s_{m}\left(t-\frac{d}{c}(m \sin \phi \cos \theta+n \sin \phi \sin \theta)\right),
$$

where $\alpha=\alpha_{1} \alpha_{2}$. We assume, $\phi \approx 90^{\circ}$ [4]. The signal is then down converted by $\Omega_{m}$ to get the output $y_{n, m}(t)$, which, after sampling at sampling intervals $T_{s}$, is, in the frequency domain

$$
Y_{n, m}\left(e^{j \omega}, \theta\right)=\alpha e^{-j \frac{d}{c T_{s}}\left(\omega+\omega_{m}\right)(m \cos \theta+n \sin \theta)} \tilde{S}\left(e^{j \omega}\right)
$$

where $\omega_{m}=\Omega_{m} T_{s}$. The similarity of above expression with (1) motivates us to use constant multipliers $w_{n, m}$ to process the signals $y_{n, m}(k)$ as done in Section II. However, the additional multiplying term $e^{-j \omega_{m} \frac{d}{c T_{s}}(m \cos \theta+n \sin \theta)}$ due to the $m$ th carrier (which however does not depend on the frequency $\omega$ ) makes the determination of the weights $w_{n, m}$ different from that in Section II.

Suppose we wish to produce a desired (frequency invariant) beampattern $B(\theta)$ by multiplying the signals $y_{n, m}(k)$ with constant multipliers $w_{n, m}$ and then adding them up. Then the processed output $y(t)$ can be described in the frequency domain as

$Y\left(e^{j \omega}, \theta\right)=\alpha \sum_{m=0}^{M-1 N-1} \sum_{n=0} w_{n, m} e^{-j \frac{d}{c T_{s}}\left(\omega+\omega_{m}\right)(m \cos \theta+n \sin \theta)} \tilde{S}\left(e^{j \omega}\right)$.

Denoting

$G(\omega, \theta)=\sum_{m=0}^{M-1} \sum_{n=0}^{N-1} w_{n, m} e^{-j \frac{d}{c T_{s}}\left(\omega+\omega_{m}\right)(m \cos \theta+n \sin \theta)}$

we can write $Y\left(e^{j \omega}, \theta\right)=\alpha G(\omega, \theta) \tilde{S}\left(e^{j \omega}\right)$. We want

$$
G(\omega, \theta) \approx B(\theta), \quad \omega_{l} \leq \omega \leq \omega_{h}
$$

where $\omega_{l}=2 \pi f_{l} T_{s}, \omega_{h}=2 \pi f_{h} T_{s}$. This implies $Y\left(e^{j \omega}, \theta\right) \approx$ $\alpha B(\theta) \tilde{S}\left(e^{j \omega}\right)$. We can then pass the resulting signal through a filter matched to $\tilde{S}\left(e^{j \omega}\right)$ and sample the output at time index $k=0$, to get the final beampattern as

$$
y(\theta) \approx \alpha B(\theta) R_{s s}(0) .
$$

where $R_{s s}(l)$ is the autocorrelation of $\tilde{s}(k)$ at lag $l$. The crucial step is to realize (15) and we cannot apply the direct 2-D IDFT technique of Section II here. So instead we estimate the weights $w_{n, m}$ using the least-squares method as follows:

Sample $G(\omega, \theta)$ at $M_{1}$ frequency points $\omega_{i}$ between $\omega_{l}$ and $\omega_{h}, i=0,1, \cdots, M_{1}-1$ and corresponding to each $\omega_{i}$, sample $G\left(\omega_{i}, \theta\right)$ and $B(\theta)$ at $N_{1}$ values of $\theta_{k}$, between $-\frac{\pi}{2}$ and $\frac{\pi}{2}$, $k=0,1, \cdots, N_{1}-1$. We wish to find $w_{n, m}$ to achieve

$$
\min _{w_{n, m}} \sum_{\omega_{i}} \sum_{\theta_{k}}\left|G\left(\omega_{i}, \theta_{k}\right)-B\left(\theta_{k}\right)\right|^{2} .
$$

The Least Squares solution to the above problem is given by

$$
\mathbf{w}=\left(\mathbf{A}^{H} \mathbf{A}\right)^{-1} \mathbf{A}^{H} \mathbf{C},
$$

where the elements of the vectors $\mathbf{w}, \mathbf{C}$ and the matrix $\mathbf{A}$ are given by

$$
\begin{gathered}
\mathbf{w}_{j}=w_{n, m}, \quad \mathbf{C}_{i}=B\left(\theta_{k_{2}}\right) \\
\mathbf{A}_{i, j}=e^{-j \frac{d}{c T_{s}}\left(\omega_{k_{1}}+\omega_{m}\right)\left(m \cos \theta_{k_{2}}+n \sin \theta_{k_{2}}\right)} .
\end{gathered}
$$

Here the indices $i$ and $j$ are related to $m, n, k_{1}$ and $k_{2}$ as

$$
i=k_{1} N_{1}+k_{2}, \quad j=m N+n
$$

with $m=0,1, \cdots, M-1, n=0,1, \cdots, N-1, k_{1}=$ $0,1, \cdots, M_{1}-1, k_{2}=0,1, \cdots, N_{1}-1$. In case $\mathbf{A}$ is not full rank, we replace $\left(\mathbf{A}^{H} \mathbf{A}\right)^{-1} \mathbf{A}^{H}$ by the Moore-Penrose pseudo inverse of $\mathbf{A}$.

\section{DESIGN EXAMPLES}

We provide two examples, one for the frequency invariant beamspace MVDR beamforming for the rectangular array and the second one for the frequency invariant beampattern synthesis using the MIMO radar virtual array. 
A. Beamspace frequency invariant MVDR Beamforming without filters

Following the procedure described in Sections II and III, we provide a design example here for a rectangular array of size $M=17, N=17$. The number of beams spanning the beamspace is $P=7$. The spacings $d_{1}$ and $d_{2}$ are both chosen to be $\frac{\lambda_{\min }}{2}$ where $\lambda_{\min }$ is the lowest wavelength in the frequency band under consideration. We intend to achieve frequency invariance in the range $1000 \mathrm{~Hz}$ to $2000 \mathrm{~Hz}$. We consider two wideband jammers arriving at angles $30^{\circ}$ and $45^{\circ}$ with jammer to signal ratio $30 \mathrm{~dB}$ and signal to noise ratio $10 \mathrm{db}$. The resulting MVDR beampattern is plotted in Fig. 3,

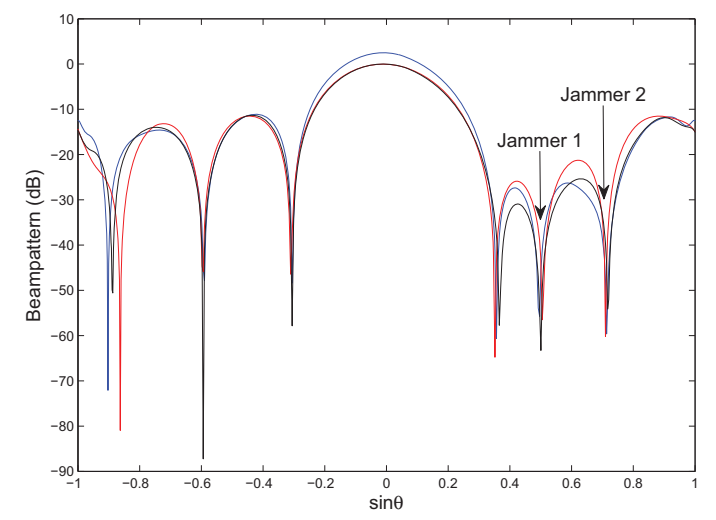

Fig. 3. MVDR Beampattern as a function of sine of azimuthal angle $\theta$ at frequencies $1000 \mathrm{~Hz}, 1500 \mathrm{~Hz}$, and $2000 \mathrm{~Hz}$.

as a function of $\sin \theta$ for frequencies $f=1000 \mathrm{~Hz}, 1500 \mathrm{~Hz}$ and $2000 \mathrm{~Hz}$. It can be seen that the shape of the mainlobe and nulls in the direction of jammers have been maintained throughout the frequency band.

\section{B. Frequency Invariant Beamforming using MIMO Radar Virtual Array}

In this example, we generate a given frequency invariant beampattern, as produced by a rectangular receiving array of size $M \times N$, with only $M=25$ transmitting antennas and $N=25$ receiving antennas as described in Section IV. We assume $f_{l}=500 \mathrm{~Hz}, f_{h}=1000 \mathrm{~Hz}, \Omega_{0}=750 \mathrm{~Hz}$, $\Omega_{m+1}=\Omega_{m}+500 \mathrm{~Hz}, m=0,1, \cdots, 23$. We sample at 25 frequency points between $500 \mathrm{~Hz}$ and $1000 \mathrm{~Hz}$ and 80 values of $\theta$ between $-\frac{\pi}{2}$ to $\frac{\pi}{2}$ to get a total of 2000 points which we use to estimate the weights using least squares technique. The desired beampattern is chosen to be $B(\theta)=\sum_{n=0}^{6} e^{-j n \pi \sin \theta}$. The synthesized pattern is plotted as a $3-\mathrm{D}$ plot with respect to frequency and azimuthal angle. The frequency invariance of the plot is clear from Fig. 4.

\section{CONCLUding Remarks}

In this paper, we performed beamspace MVDR beamforming for wideband signals using a rectangular array without any time domain filtering, by first designing a set of fixed frequency invariant beams using one multiplier at each sensor output and then combining them using a set of adaptable weights. This results in far lesser complexity compared to traditional wideband adaptive beamforming with satisfactory performance as shown by the simulation examples. We also

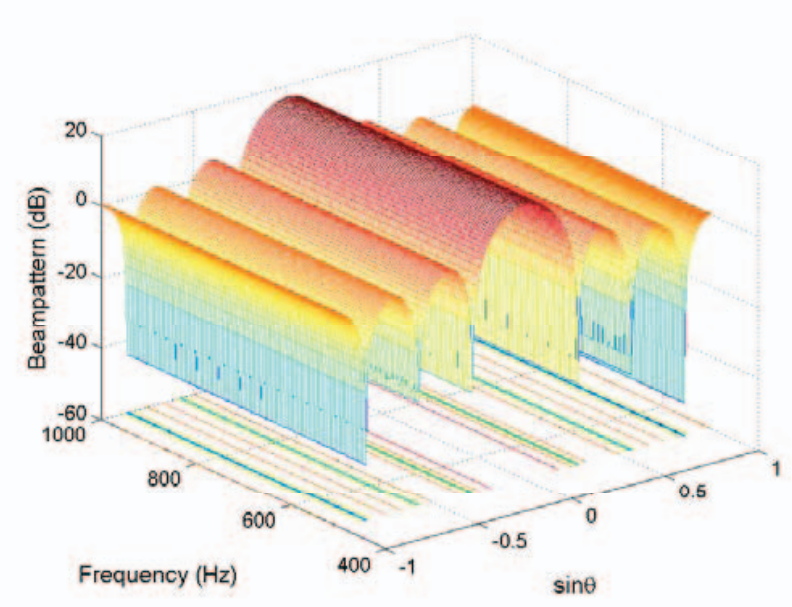

Fig. 4. Frequency invariant beam designed using MIMO radar virtual array concept.

developed a technique to design the fixed frequency invariant beams using the MIMO virtual array concept using only $M$ transmitting and $N$ receiving antennas instead of a rectangular receiving array of size $M \times N$. The simulation examples satisfactorily show the effectiveness of the proposed technique.

\section{REFERENCES}

[1] D. W. Bliss, and K.W. Forsythe, "Multiple-input multiple-output (MIMO) radar and imaging: Degrees of freedom and resolution", Proc. 37th IEEE Asilomar Conf. Signlas, Systems, Computers, Nov. 2003, vol. 1, pp. 54 59.

[2] J. Capon, "High-resolution frequency-wavenumber spectrum analysis," Proc. IEEE, vol. 57, no. 8, pp. 1408-1418, Aug. 1969.

[3] S. C. Chan, and H. H. Chen, "Uniform Concentric Circular Arrays With Frequency-Invariant Characteristics-Theory, Design, Adaptive Beamforming and DOA Estimation," IEEE Trans. on Signal Processing, vol. 55, Issue 1, pp. 165-177, Jan. 2006.

[4] M. Ghavami, "Wideband smart antenna theory using rectangular array structures," Proc. IEEE Trans. on Signal Processing, vol. 50, Issue 9 ,

[5] M. Ghavami, "An adaptive wideband array using a single real multiplier for each antenna element," The 13th IEEE International Symposium on Personal, Indoor and Mobile Radio Communications, 2002, vol. 4, pp. 1805-1809, Sept. 2002.

[6] B. Liu, C. Han, and B. Liu, "Receiving Signal Processing of Wideband MIMO Radar Based On Transmitting Diversity," International Conference on Radar, 2006, CIE '06, pp. 1-4, Oct. 2006.

[7] W. Liu, D. McLernon, and M. Ghogho, "Frequency Invariant Beamforming Without Tapped Delay-Lines," IEEE International Conference on Acoustics, Speech and Signal Processing (ICASSP), 2007, vol. 2, pp. II-997 - II-1000, Apr. 2007.

[8] W. Liu, R. Wu, and R. J. Langley, "Design and Analysis of Broadband Beamspace Adaptive Arrays," IEEE Trans. on Antennas and Propagation, vol. 55, Issue 12, pp. 3413-3420, Dec. 2007.

[9] T. Sekiguchi, and Y. Karasawa, "Wideband beamspace adaptive array utilizing FIR fan filters for multibeam forming," IEEE Trans. on Signal Processing, vol. 48, Issue 1, pp. 277-284, Jan. 2000.

[10] M. Uthansakul, and M. E. Bialkowski, "Fully spatial wide-band beamforming using a rectangular array of planar monopoles," IEEE Trans. on Antennas and Propagation, vol. 54, Issue 2, Part 2, pp. 527-533, Feb. Anten. 2006 .

[11] D. B. Ward, Z. Ding, and R. A. Kennedy, "Broadband DOA Estimation using frequency invariant beamforming," IEEE Trans. on Signal Processing, vol. 46,no. 5, pp. 1463-1469, May 1998.

[12] H. L. Van Trees, Optimum Array Processing: Part IV of Detection, Estimation and Modulation Theory. New York: Wiley Interscience, 2002. 